\title{
The Plane's Diving Maneuvering Characteristics Simulation in Vertical Surface
}

\author{
Jia Zhonghu \\ Department of Airborne Vehicle Engineering of Naval \\ Aeronautical and Astronautical University \\ Yantai, China \\ Jiazh586@sina.com
}

\section{Liu Wenlin}

Department of Airborne Vehicle Engineering of Naval

Aeronautical and Astronautical University

Yantai, China

Liuwl@sina.com

Tao Yang

Department of Airborne Vehicle Engineering of Naval Aeronautical and Astronautical University

Yantai, China

Taoyang@sina.com

\begin{abstract}
The characteristics of flight dynamics in each diving section are analyzed, and then the dynamic models are built. After simulating the plane's diving maneuvering flight, the parameters changing curves with and without external stores are plotted. And the results could provide theoretical basis to improve the plane's maneuvering performance.
\end{abstract}

Keywords: vertical surface; diving; flight simulation; maneuvering characteristics; maneuvering performance

\section{INTRODUCTION}

Diving is the common motion of tactical flight[1][2], and can speed up the plane in a very short time. The flight path can be divided into three parts: curved section of entering diving, straight section of diving and the curved section of exiting diving. Since the altitude would lose rapidly when diving, in most cases the time of diving won't last long. During this time, entering and exiting diving are a large portion. Thus, the performances calculating make a big difference.

\section{CENTRIPETAL FORCE DURING DIVING}

The direction of centripetal force when entering diving is straight down the tangent line of flight path, while exiting diving the direction is straight up. The centripetal force when entering diving is:

\author{
Wang Yunliang \\ Department of Airborne Vehicle Engineering of Naval \\ Aeronautical and Astronautical University \\ Yantai, China \\ Wangyl@sina.com \\ Gao Yong \\ Department of Airborne Vehicle Engineering of Naval \\ Aeronautical and Astronautical University \\ Yantai, China \\ Gaoyong@sina.com
}

$$
F_{\mathrm{x}}=G \cos \theta-Y
$$

The centripetal force when exiting diving is:

$$
F_{\mathrm{x}}=Y-G \cos \theta
$$

\section{CALCULATING MODEL OF ENTERING DIVING}

The plane's altitude decreases with the velocity increasing rapidly when entering diving. When exiting diving the plane's velocity increases less. Obviously, the bigger the centripetal force is the smaller the radius of flight path is.

From Eq.(1)

$$
F_{\mathrm{x}}=\frac{m V^{2}}{r}=G \cos \theta-Y
$$

So

$$
r=\frac{m V^{2}}{G \cos \theta-Y}=\frac{V^{2}}{g\left(\cos \theta-n_{y}\right)}
$$

Rewrite the acceleration, where $d L$ stands for the unit length,

$$
\frac{d V}{d t}=\frac{d V^{2}}{2 d L}=\frac{d V^{2}}{2 r d \theta}
$$


When entering diving, the plane's movement equations are[3]

$$
\left\{\begin{array}{l}
m \frac{d V}{d t}=P \cos \left(\alpha+\varphi_{P}\right)-Q-G \sin \theta \\
-m V \frac{d \theta}{d t}=-P \sin \left(\alpha+\varphi_{P}\right)-Y+G \cos \theta
\end{array}\right.
$$

(6)

The first formula in Eq.(6) is simplified into

$$
m \frac{d V}{d t}=P+G \sin \theta-Q
$$

Combing Eq.(5) and Eq.(7):

$$
m \frac{d V^{2}}{2 r d \theta}=P+G \sin \theta-Q
$$

Then

$$
\Delta V^{2}=\frac{2 r g}{G}(P+G \sin \theta-Q) \Delta \theta
$$

From Eq.(9), a $\Delta V_{1}^{2}$ can be got. When certify $\Delta V$, $Q=C_{x} S \frac{\rho V_{1}^{2}}{2}$, where $V_{1}$ is the initial velocity when entering diving. If the plane levels off before diving, the projective shadow of gravity at the tangent line of flight path is equal to $G \sin \frac{\Delta \theta}{2}$.

After calculating $\Delta V_{1}^{2}$, supposing the square of velocity is $\Delta V_{1}^{2}+V_{1}^{2}, r_{2}$ can be got from the formula below[4]

$$
r_{2}=\frac{V_{1}^{2}+\Delta V_{1}^{2}}{g\left(\cos \theta-n_{y}\right)}
$$

Then $\Delta V_{2}^{2}$ can be got from Eq.(9). Obviously, after the flight path deflects $2 \Delta \theta$, the square of velocity is $\Delta V_{2}^{2}+\Delta V_{1}^{2}+V_{1}^{2}$. Then find $r_{3}$ and $\Delta V_{3}^{3}$. Repetitive computation until $\sum \Delta \theta$ is equal to slant angle of flight path. The changing of velocity when entering diving can be got from the increment of velocity $\Delta V_{2}^{2}+\Delta V_{1}^{2}+V_{1}^{2}+\cdots \cdots$

The time of entering diving is

$$
\sum \Delta t=\frac{r_{1} \Delta \theta}{V_{1}}+\frac{r_{2} \Delta \theta}{\sqrt{V^{2}+\Delta V_{1}^{2}}}+\frac{r_{3} \Delta \theta}{\sqrt{V^{2}+\Delta V_{1}^{2}+\Delta V_{2}^{2}}}+\cdots
$$

\section{CALCULATING MODEL OF STRAIGHT SECTION} OF DIVING

When during the straight section of diving, $\frac{d \theta}{d t}=0$,combine Eq.(6), the simplified equations are[4]

$$
\left\{\begin{array}{l}
\frac{d V}{d t}=\frac{g}{G}(P-Q-G \sin \theta) \\
Y=G \cos \theta
\end{array}\right.
$$

The limit velocity of diving is

$$
V_{d}=\sqrt{\frac{2(P-G \sin \theta)}{C_{x} \rho S}}
$$

Often, the diving velocity is close to the limit velocity of diving, and the resistance increases rapidly. At the moment, the acceleration of diving decreases and the accelerated performance of diving become worse.

The diving velocity vs. flight altitude is[4]

$$
\frac{d V}{d t}=\frac{d V}{d H} \frac{d H}{d t}=V \sin \theta \frac{d V}{d H}
$$

And

$$
\frac{d V}{d H}=-\frac{g}{V} \frac{P-C_{x} \frac{1}{2} \rho V^{2} S}{G \sin \theta}
$$

\section{CALCULATING MODEL OF EXITING DIVING}

The loss of altitude is a important target of diving performances. When exiting diving, the pilot would pull rod to increase attack angle and let the plane's flight path curve up. When the path is close to horizontal, the pilot pushes rod to decrease attack angle. The movement equations are[4]

$$
\left\{\begin{array}{l}
\frac{d V}{d t}=-g \sin \theta \\
\frac{d \theta}{d t}=\frac{g}{V}\left(n_{y}-\cos \theta\right)
\end{array}\right.
$$

From Eq.(14)

$$
\frac{d V}{d \theta}=-\frac{V \sin \theta}{n_{y}-\cos \theta}
$$

the velocity and the slant angle of flight path are $V_{1}$ and $\theta_{1}$ when exiting diving, while they are $V$ and $\theta$ when finishing diving. Where, $\theta=0, n_{y}$ is constant [4]

$$
V=\frac{n_{y}-\cos \theta_{1}}{n_{y}-1} V_{1}
$$

And

$$
\Delta H=\frac{1}{2 g}\left(V^{2}-V_{1}^{2}\right)
$$

The loss of altitude is

$$
\Delta H=\frac{V_{1}^{2}}{2 g}\left[\left(\frac{n_{y}-\cos \theta_{1}}{n_{y}-1}\right)^{2}-1\right]
$$

\section{SIMULATION}


The state of engine keeps unchangeable in each flight section in simulation. The initial conditions are: the diving angle $\theta=-30^{\circ}$, the initial diving altitude $7000 \mathrm{~m}$, the initial diving velocity $\mathrm{V} 0=300 \mathrm{~m} / \mathrm{s}$. And the simulated curves are shown in Fig.1 4.

It is shown that the bigger the plane's velocity is, the more the loss of altitude is. When the plane takes mounts, the resistance coefficient increases, the loss of plane's energy grows bigger. So the capability of acceleration gets worse, and the changing process of altitude becomes slower.
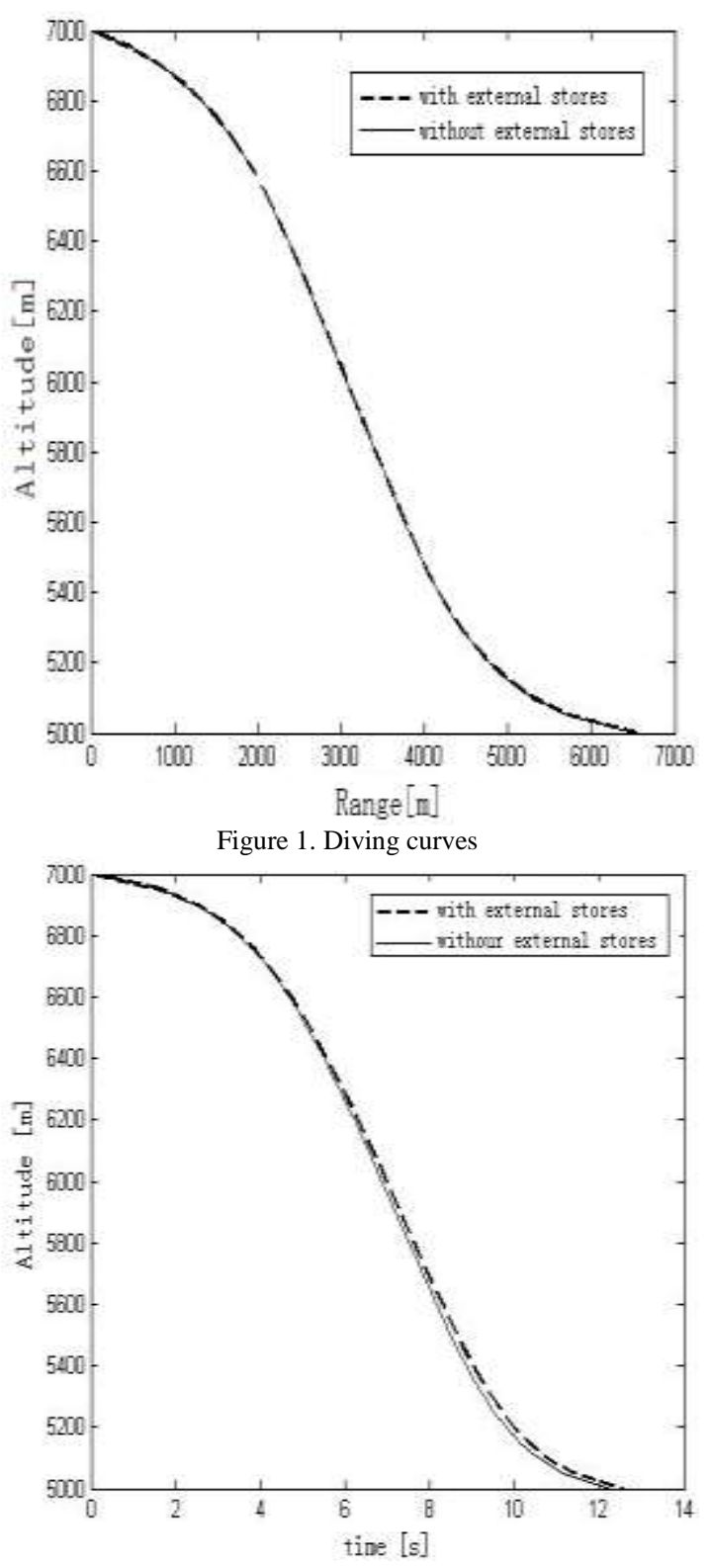

Figure 2. Altitude vs. time

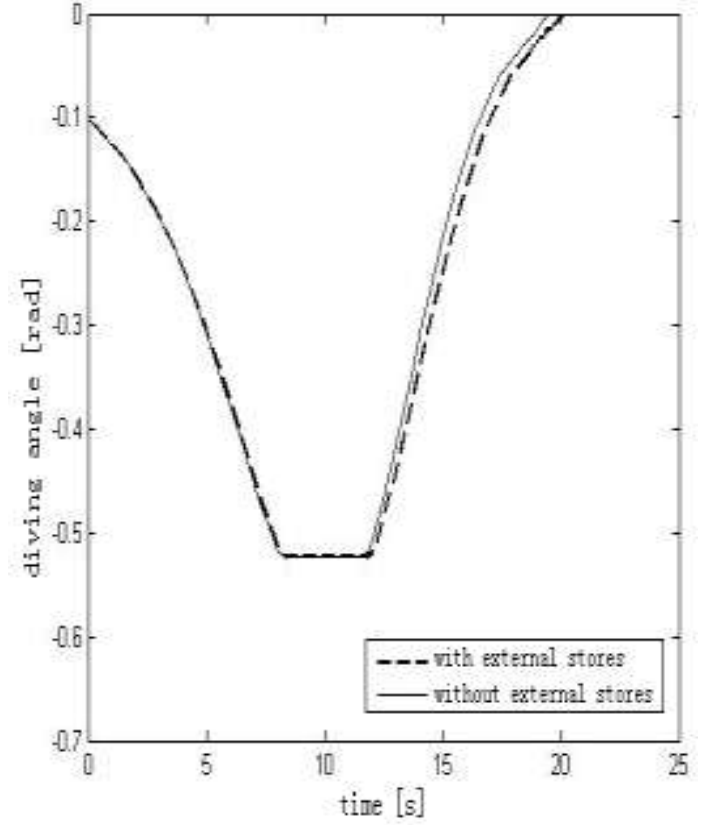

Figure 3. Diving angle vs. time

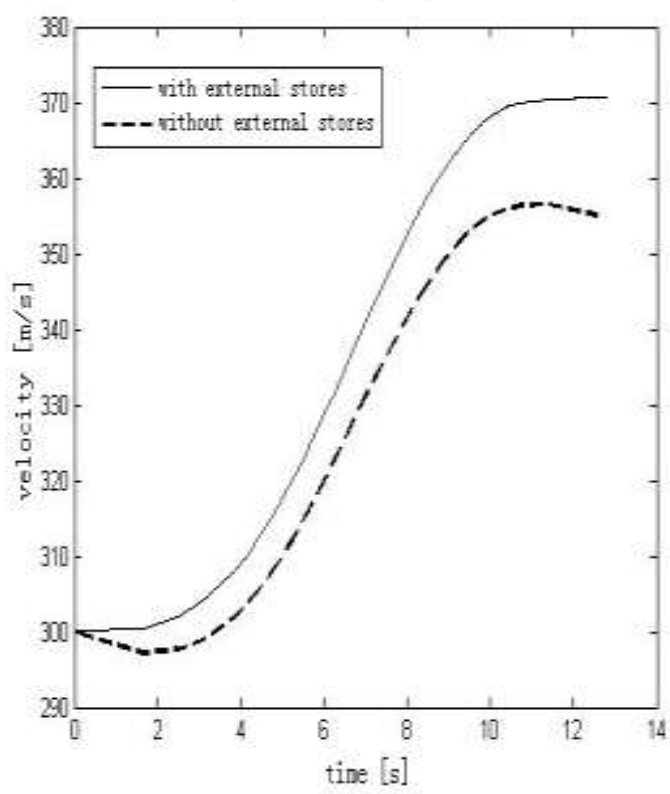

Figure.4 Velocity vs. time

\section{CONCLUSION}

The diving maneuvering flight in vertical surface is discussed, the flight dynamics models of each diving section are built. Compared the different conditions between with and without external stores, the changes of diving performance are reflected. The results could provide theoretical basis to improve the plane's maneuvering performance.

\section{REFERENCES}


[1] Gao H, Zhu $\mathrm{P}$ K, Gao Z H. The Advanced Flight Dynamics[M].Beijing: National Defence Industry Press, 2004: 3 5.

[2] Wang X R. The Technology of Flight Simulation[J]. Technology of Test and Control, 1994, 13(2): 47 50.

[3] Liu D G, Fei J G. Numerical Simulation Algorithm of Dynamics System [M]. Beijing: Science Press, 2000: 15 20.

[4] McQuay W K. Distributed Collaborative Environments for 21st Century Modeling

Simulation[J] .Simulation.2001,76(2):94 95

[5] Xu D Q. Flight Dynamics[M]. Beijing: Tide Press, 2010: 174 178.

[6] Fang Z P, Chen W C, Zhang S G. Flight Dynamics of Aircraft[M]. Beijing: Beijing University of Aeronautical and Astronautical Press, 2005: 95 102.

[7] Cook M V. Flight Dynamics Principles[M]. Elsevier Ltd, 2007: 66-79.

[8] Torenbeek E. Wittenberg H. Flight Physics[M]. Springer Science+Business Media, 2009: 314-319.

[9] Hull D G. Fundamentals of Airplane Flight Mechanics[M]. Springer-Verlag Berlin Heidelberg, 2007: 79-106.

[10] Etkin B. Dynamics of Atmospheric Flight[M]. John Wiley \& Sons, 1nc. 1972: 129-145. 
